\title{
Ursolic acid protects against ulcerative colitis via anti-inflammatory and antioxidant effects in mice
}

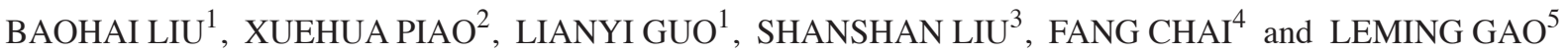 \\ Departments of ${ }^{1}$ Gastroenterology, ${ }^{2}$ Traditional Chinese Medicine, ${ }^{3}$ Clinical Laboratory and ${ }^{4}$ General Surgery, \\ The First Affiliated Hospital, Liaoning Medical University, Jinzhou, Liaoning 121001; \\ ${ }^{5}$ The 2nd Clinic, Stomatology Hospital, Peking University, Beijing 100101, P.R. China
}

Received April 10, 2015; Accepted February 16, 2016

DOI: $10.3892 / \mathrm{mmr} .2016 .5094$

\begin{abstract}
Ursolic acid (UA) has been reported to have a protective effect in colitis. However, the underlying mechanisms remain to be elucidated. In the present study, experimental ulcerative colitis was induced in male BALB/c mice by the administration of $5 \%$ dextran sulfate sodium (DSS) for 7 days, followed by treatment with UA for another 7 days. Hematoxylin \& eosin staining was performed to evaluate colon tissue damage, and enzyme assays were used to measure malondialdehyde (MDA) content and superoxide dismutase (SOD) activity in colon homogenate. In addition, serum levels of interleukin (IL)-1 $\beta$ and tumor necrosis factor (TNF)- $\alpha$ were measured using an ELISA, and the level of nuclear factor (NF) $-\kappa \mathrm{B}$ p65 in the colonic tissues was assessed by western blotting. The 7-day DSS administration induced marked colon damage, increased the serum levels of IL-1 $\beta$ and TNF- $\alpha$, increased MDA content and decreased SOD activity in the colon homogenate. These changes were significantly improved by treatment with UA. UA also reduced the DSS-stimulated high nuclear level of NF- $\kappa \mathrm{B}$ p65 in the colon tissues. These results demonstrate a protective role of UA in ulcerative colitis, and suggest that anti-inflammatory and antioxidant activities are involved in the underlying mechanisms.
\end{abstract}

\section{Introduction}

Ulcerative colitis, a type of inflammatory bowel disease, is characterized by uncontrolled inflammation in the colon and rectum, recurrent episodes of bloody diarrhea, cramping, abdominal pain, mucosal inflammation and injury (1). Considering the pathogenic mechanisms of ulcerative colitis, it is suggested that deregulation of the pro-/anti-inflammatory

Correspondence to: Dr Xuehua Piao, Department of Traditional Chinese Medicine, The First Affiliated Hospital, Liaoning Medical University, 2 Renmin Street, Jinzhou, Liaoning 121001, P.R. China E-mail: piaoxuehua82@gmail.com

Key words: anti-inflammatory, antioxidant, malondialdehyde, superoxide dismutase, ulcerative colitis, ursolic acid systems and antioxidant system may to be involved (2). Currently, the medical treatment of ulcerative colitis relies predominantly on the use of traditional drugs, including aminosalicylates, corticosteroids and immunosuppressants. These conventional therapies for ulcerative colitis fail to successfully induce remission or prevent relapse, and can also cause various side effects (3). Therefore, there is an increasing interest in identifying alternative and more tolerable treatments for this disease.

Ursolic acid (UA), a natural pentacyclic triterpenoid carboxylic acid, is abundant in numerous plants, including apples, blueberries and hawthorn berries, and is the major component of certain oriental medicinal herbs, which are widely used in folk medicine (4). UA is well known to possess various biological activities, including anti-inflammatory $(5,6)$, anticancer $(7,8)$, hypoglycemic (9), antioxidant (10-12) and immunomodulatory activities (13). Previously, UA was reported to be involved in colitis in a number of publications $(4,14,15)$. Hawthorn berry extract containing $0.5 \%$ UA decreased edema and the infiltration of neutrophils in the colonic tissues of acetic acid-treated rats (14). UA significantly inhibits the production of pro-inflammatory cytokines, including tumor necrosis factor (TNF)- $\alpha$, interleukin (IL)-1 $\beta$ and IL-6, and the expression of cyclooxygenase-2 and inducible nitric oxide (NO) synthetase in lipopolysacharride (LPS)-stimulated macrophages and colonic tissues from 2,4,6-trinitrobenzenesulfonic-treated mice $(4,15)$. UA also exerts an inhibitory effect on the activation of nuclear factor (NF) $-\kappa \mathrm{B}$ in human intestinal epithelial cells and macrophages $(4,15)$. These results indicate a protective role of UA in colitis. However, the detailed underlying mechanisms remain to be fully elucidated.

The present study aimed to investigate the protective effect of UA in a dextran sulfate sodium (DSS)-induced mouse model of ulcerative colitis, a widely used ulcerative colitis model (16), in order to elucidate the potential mechanisms underlying the anti-inflammatory and antioxidative activities of UA.

\section{Materials and methods}

Reagents. UA was purchased from Shanxi Huike Plant Development Co., Ltd. (Xi'an, China; purity $\geq 98 \%$ ), dissolved in dimethyl-sulphoxide (DMSO; Sigma-Aldrich, St. Louis, MO, USA), and diluted in normal saline solution. The total 
quantity of DMSO did not exceed 1\% upon assessment; a dose considered of no significance in the assays used. DSS was obtained from MP Biomedicals (Solon, OH, USA) and dissolved in distilled water. Chemiluminescence reagent was purchased from Pierce (Rockford, IL, USA). Rabbit anti-NF- $\kappa$ B p65 monoclonal antibody (cat. no. 4764) and rabbit anti- $\beta$-actin polyclonal antibody (cat. no. 4967) were purchased from Cell Signaling Technology, Inc. (Danvers, MA, USA). Horseradish peroxidase (HRP)-conjugated goat anti-rabbit immunoglobulin (Ig)G secondary antibody (cat. no. sc-2004) was purchased from Santa Cruz Biotechnology, Inc. (Dallas, Texas, USA).

Animals. A total of 36 male BALB/c mice (7-week-old; 18-22 g) were obtained from the Experiment Animal Center, Liaoning Medical University (Jinzhou, China). The mice were allowed to adapt to the laboratory environment for 1 week prior to experiments. The animals were housed in plastic cages containing corn chip bedding, and were maintained on a 12-h light-dark cycle (07:00-19:00 h light cycle; 19:00-07:00 h dark cycle) with a room temperature of $22 \pm 1^{\circ} \mathrm{C}$ and a humidity of $65-70 \%$. Water and food were available ad libitum. The animal experiments were approved by the Ethical and Research Committee of Liaoning Medical University (Jinzhou, China).

Induction of ulcerative colitis and UA treatment. DSS was used to induce ulcerative colitis in the present study. DSS-induced colitis is characterized by the mucosal infiltration of inflammatory cells, epithelial injury and ulceration, which are similar to acute and chronic ulcerative colitis in humans (17). In the present study, the mice were divided into four groups, as follows (9 mice/group): Control, DSS, DSS+UA and DSS+sulfasalazine [SFZ; a reference drug in colitis treatment (2)]. Colitis was induced by the provision of distilled water containing 5\% DSS (w/v) for 7 days ad libitum, followed by fresh water. The mice in the DSS+UA and DSS+SFZ groups were then treated with UA $(20 \mathrm{mg} / \mathrm{kg})$ or SFZ $(100 \mathrm{mg} / \mathrm{kg})$, respectively, by gavage once per day for another 7 days. The mice in the DSS group were provided with the same volume of saline by gavage. The mice were assessed daily for behavior, body weight, presence of blood in stools and stool consistency.

Evaluation of disease activity index (DAI). The DAI was calculated for each animal, as reported previously (18). Briefly, the DAI was determined by combining the scores assigned for body weight loss, stool consistency and stool blood, with the mean of the three values deemed the DAI. A higher DAI score is indicative of more severe colon damage. Body weight loss was calculated as the percentage difference between the initial body weight and the final body weight. Stool blood was determined using the Fecal Occult Blood Test kit (Baso Diagnostics Inc., Zhuhai, China). The parameters investigated for the DAI evaluation are listed in Table I.

Measurement of serum $I L-1 \beta$ and TNF- $\alpha$. Blood samples $(0.5 \mathrm{ml} / \mathrm{mouse})$ were collected from all mice following anesthetization with $0.1 \mathrm{ml}$ phenobarbital (Sigma-Aldrich) and sacrifice by decapitation. Serum was collected following centrifugation at $2,000 \times \mathrm{g}$ for $10 \mathrm{~min}$ at $4^{\circ} \mathrm{C}$ and aliquoted. The aliquots $(150 \mu \mathrm{l})$ were stored at $-20^{\circ} \mathrm{C}$ until use. The serum levels of IL- $1 \beta$ and TNF- $\alpha$ were measured using ELISA kits (cat. nos. EK0394 and EK0527, respectively; Wuhan Boster Biological Engineering Co., Ltd., Wuhan, China), according to the manufacturer's protocol.

Assessment of pathological damage. For histological analysis, the colons following collection of blood samples and rinsed with phosphate-buffered saline (PBS; pH 7.4). From each colon, two 2-cm long segments were removed from the proximal and distal ends, which were fixed in $4 \%$ paraformaldehyde (Sigma-Aldrich), dehydrated and embedded in paraffin (Sigma-Aldrich). These colon segments were cut longitudinally into $4-\mu \mathrm{m}$ thick sections, which were then stained with hematoxylin and eosin (H\&E; Leica Microsystems, Inc., Buffalo Grove, IL, USA). The colon sections were interpreted according to the criteria listed in Table II. The final colon pathological score was calculated as the average of the scores from the proximal and distal segments of the mouse colons.

Measurement of malondialdehyde (MDA) content, and superoxide dismutase (SOD) and myeloperoxidase (MPO) activities. The transverse colon tissues were rinsed and weighed, and were then placed into tubes with nine volumes of normal saline. The tissue samples were then homogenized. Following centrifugation at $3,000 \mathrm{x}$ for $10 \mathrm{~min}$ at $4^{\circ} \mathrm{C}$, the MDA content, and activities of SOD and MPO in the supernatant, were measured using commercially available kits (cat. nos. A003, A001 and A044, respectively; Nanjing Jiancheng Bioengineering Institute, Nanjing, China), according to the manufacturer's protocol.

Western blotting for the protein expression of $N F-\kappa B$ p 65 . The nuclear proteins in the transverse colon tissues were extracted using a kit, according to the manufacturer's protocol (Wuhan Boster Biological Engineering Co., Ltd.). The protein concentration was quantified using a bicinchoninic acid assay (Thermo Fisher Scientific, Inc., Waltham, MA, USA). The nuclear protein expression of $\mathrm{NF}-\kappa \mathrm{B}$ p65 was measured using Western blotting, in accordance with a previous report (19). Briefly, the nuclear proteins $(20 \mu \mathrm{g})$ were separated electrophoretically on 4-12\% SDS-PAGE gels, and transferred onto nitrocellulose membranes (Thermo Fisher Scientific, Inc.). Following $30 \mathrm{~min}$ of blocking with $2.5 \%$ nonfat milk, the membranes were incubated with rabbit anti-NF- $\mathrm{B}$ p 65 monoclonal antibody $(1: 1,000)$ and rabbit anti- $\beta$-actin polyclonal antibody $(1: 2,000)$ at $4^{\circ} \mathrm{C}$ overnight, followed by $1 \mathrm{~h}$ incubation with HRP-conjugated goat anti-rabbit IgG $(1: 2,000)$. The membranes were washed with PBS containing $0.5 \%$ Tween 20 (Sigma-Aldrich) subsequent to each antibody treatment. The membranes were developed using chemiluminescence reagent and then exposed to X-ray film (Midwest Scientific, Valley Park, MO, USA). The band intensities were analyzed using ImageJ software, version 1.5g (https://imagej.nih.gov/ij/). The protein levels of p65 are expressed as the ratio of the band optical intensity to that of $\beta$-actin.

Statistical analysis. Data are presented as the mean \pm standard error of the mean. Statistical analysis was performed using SPSS 17.0 statistical software (SPSS, Inc., Chicago, IL, USA). One-way analysis of variance and Dunnett's test were used 
Table I. Parameters of disease activity index evaluation.

\begin{tabular}{lclc}
\hline Score & Weight loss $(\%)$ & Stool consistency & Blood in stool \\
\hline 0 & - & Normal & - \\
1 & $1-5$ & Normal & + \\
2 & $6-10$ & Very soft but formed & ++ \\
3 & $11-15$ & Liquid & +++ \\
4 & $>15$ & Liquid & Gross rectal bleeding \\
\hline
\end{tabular}

Table II. Pathological scores of colitis in colon tissues.

\begin{tabular}{ll}
\hline Score & \multicolumn{1}{c}{ Pathological parameter } \\
\hline 0 & Normal tissues \\
1 & Mild inflammation in the mucosa with some infiltrating mononuclear cells \\
2 & Increased level of inflammation in the mucosa with more infiltrating cells, crypt \\
& glands pulled away from basement membrane, mucin depletion from goblet \\
& cells, epithelium beginning to pull away from mucosa into lumen \\
3 & Extensive infiltrating cells in the mucosa and submucosa area, crypt \\
& abscesses present, increased mucin depletion, epithelial cell disruption \\
& Extensive infiltrating cells in the tissue, complete loss of crypts \\
\hline
\end{tabular}

A

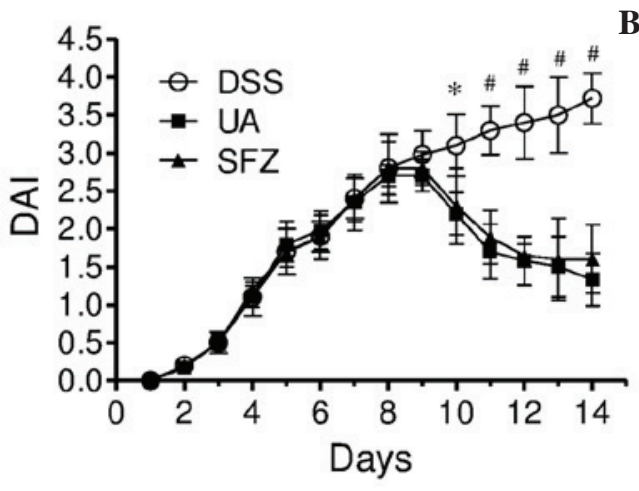

B

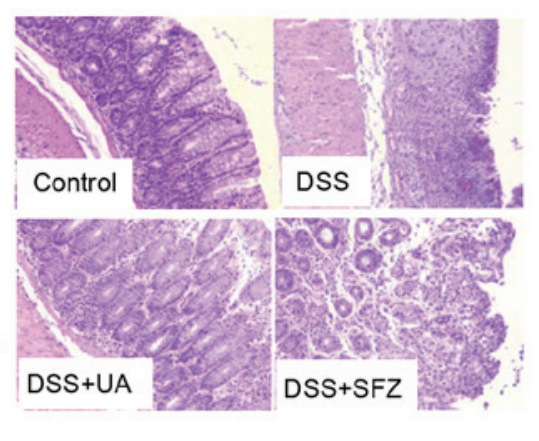

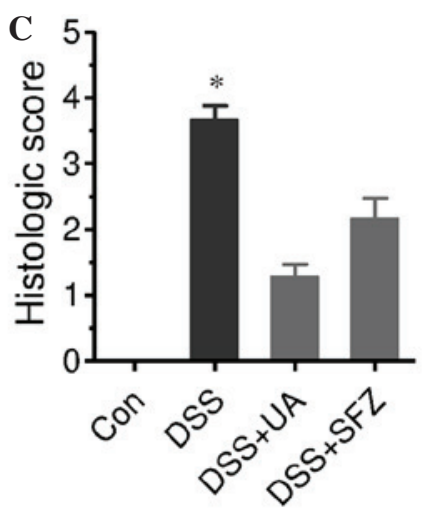

Figure 1. Effects of UA on DAI and histological injury. (A) Effects of UA on DAI. Data are presented as the mean \pm standard error of the mean. "P<0.05, ${ }^{\#} \mathrm{P}<0.01$ vs. the UA or SFZ group. (B) Histological changes in the colon were detected following hematoxylin and eosin staining (magnification, $\mathrm{x} 200$ ). (C) Pathological scores following administration of DSS for 7 days, followed by treatment with UA (20 mg $/ \mathrm{kg}$ ) or SFZ (100 mg/kg) for $7 \mathrm{days}(\mathrm{n}=6-9)$. Data are presented as the mean \pm standard error of the mean. ${ }^{\text {P }}<0.05$ vs. the control, UA and SFZ groups. DSS, dextran sulfate sodium; UA, ursolic acid SFZ, sulfasalazine; DAI, disease activity index.

for group comparisons. $\mathrm{P}<0.05$ was considered to indicate a statistically different difference.

\section{Results}

Effects of UA on DAI and histological injury. The administration of 5\% DSS in mice for 7 days resulted in marked clinical and histological signs of colitis. These mice exhibited loose stools or diarrhea, occult or gross rectal bleeding, and weight loss. The DAI score of colitis in the DSS-treated mice was significantly increased with time, and increased continuously, even following the termination of DSS treatment (Fig. 1A). Following 7 days of DSS administration, the mice were treated with UA ( $20 \mathrm{mg} / \mathrm{kg}$ daily) for another 7 days. UA treatment significantly reduced DSS-induced DAI following 3 days of treatment with UA (day 10), and this reduction was more marked following 7 days of treatment $(\mathrm{P}<0.05$; Fig 1A). SFZ, a well-known prodrug, which releases 5-aminosalicylic acid in the intestine to disturb the prostaglandin and leukotriene pathways, has been the most widely-prescribed anti-colitis drug (2). As a reference drug, SFZ (100 mg/kg daily) also significantly decreased the DSS-induced DAI in the present study $(\mathrm{P}<0.05$; Fig $1 \mathrm{~A})$.

The administration of DSS in the mice for 7 days caused marked colon tissue damage. H\&E staining of the colon tissues exhibited extensive infiltrating cells, epithelial cell disruption 

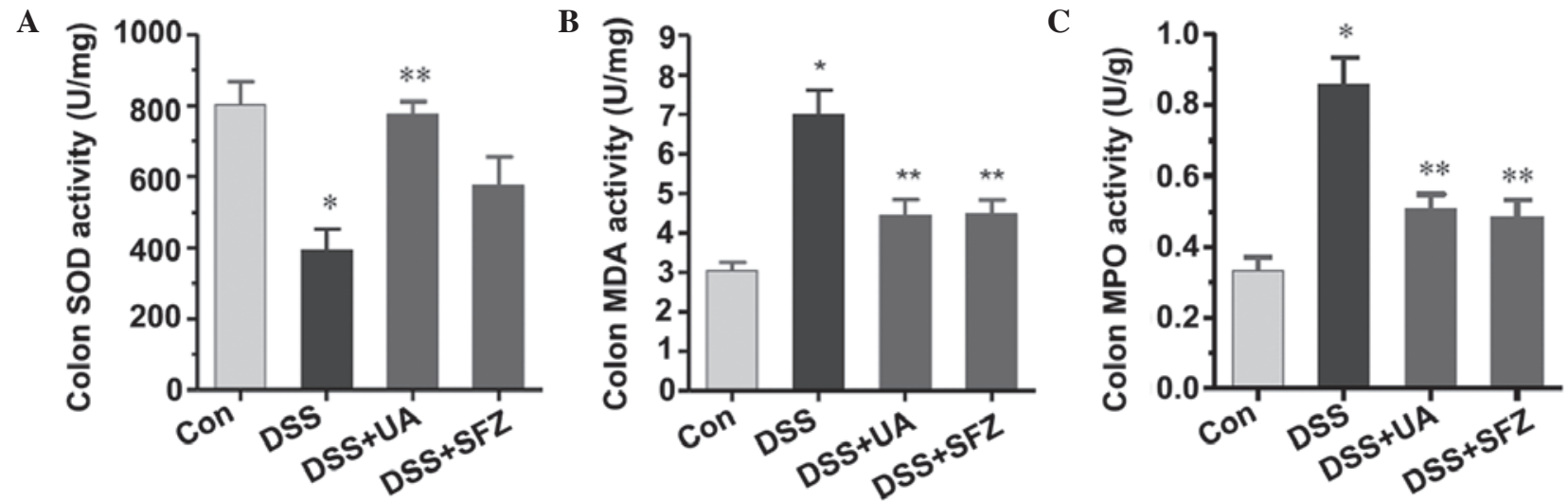

Figure 2. Effects of UA on MDA content, and SOD and MPO activities in colon tissues. The mice were treated with DSS for 7 days, and then with UA or SFZ for a further 7 days. Colon homogenates were used to measure (A) SOD activity, (B) MDA content and (C) MPO activity. The results are expressed as MDA content, and SOD and MPO activity per mg colon tissue, as the mean \pm standard error of the mean ( $\mathrm{n}=6-9)$. ${ }^{*} \mathrm{P}<0.05$, vs. Con group; ${ }^{* *} \mathrm{P}<0.05$, vs. DSS group. UA, ursolic acid; DSS, dextran sulfate sodium; SFZ, sulfasalazine; MDA, malondialdehyde; SOD, superoxide dismutase; MPO; myeloperoxidase.
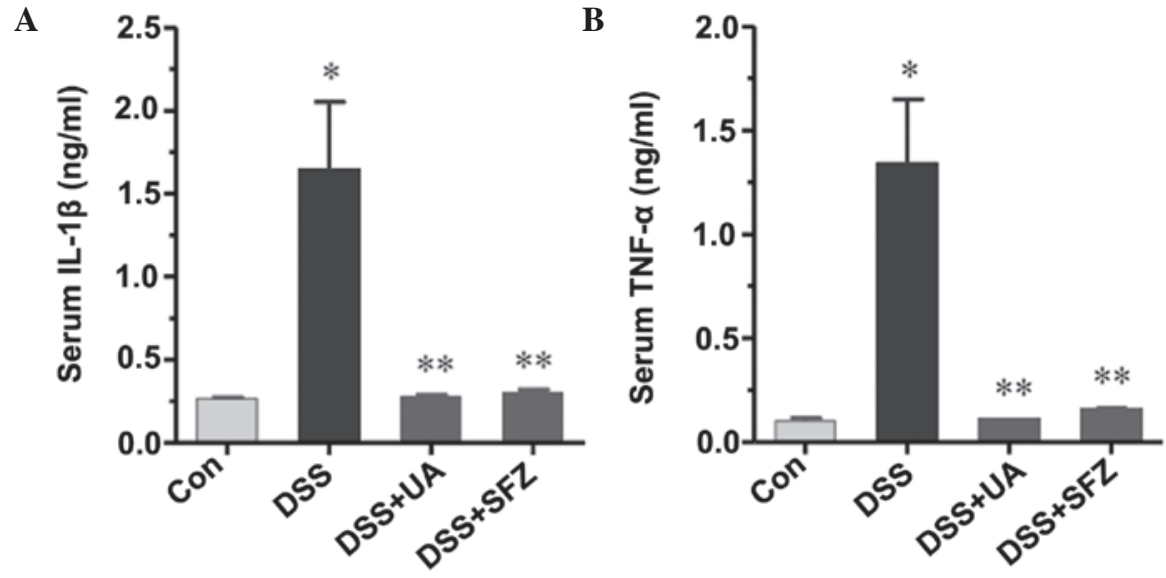

Figure 3. Effects of UA on serum levels of IL-1 $\beta$ and TNF- $\alpha$. The mice were treated with DSS for 7 days and then with UA or SFZ for a further 7 days. The serum levels of (A) IL-1 $\beta$ and (B) TNF- $\alpha$ were measured using an enzyme-linked immunosorbent assay. Data are presented as the mean \pm standard error of the mean ( $\mathrm{n}=6-9)$. "P<0.01, vs. Con group; "* $\mathrm{P}<0.05$, vs. DSS group. UA, ursolic acid; DSS, dextran sulfate sodium; SFZ, sulfasalazine; Con, control; IL-1 $\beta$, interleukin-1 $\beta$; TNF- $\alpha$, tumor necrosis factor- $\alpha$.

or the complete loss of crypts in the DSS group (Fig. 1B). In the DSS group, the histopathological score was increased to 3.67, which was significantly increased, as compared with the score of 0 in the control group $(\mathrm{P}<0.05$; Fig. 1C). UA treatment significantly improved the DSS-induced colon histological injury, and decreased the pathological score to $1.29(\mathrm{P}<0.05)$. The reference drug, SFZ, also improved histological injury, with a pathological score of 2.17 ( $\mathrm{P}<0.05$, vs. DSS group). The results from the DAI and pathological examinations demonstrated the protective role of UA in colitis.

UA decreases MDA content and MPO activity, and increases SOD activity in colon tissues. The present study then examined the possible mechanisms by which UA offers protection from colitis. As UA exhibits antioxidant activity in certain cell models or cell-free models (10-12), the present study aimed to determine whether UA interferes in antioxidant ability in colon tissues. The content of MDA, a marker of lipid peroxidative damage, and activity of SOD, an important anti-oxidative enzyme were measured. As shown in Fig. 2A and B, DSS administration significantly decreased SOD activity and increased MDA content in the colon tissues, compared with the control group $(\mathrm{P}<0.05)$. Treatment with UA significantly reversed the DSS-induced changes in SOD activity and MDA content $(\mathrm{P}<0.05)$. SFZ also induced a significant decrease in DSS-stimulated MDA content, and a non-significant increase in SOD activity. Hawthorn berry extract, a source of UA, has been found to decrease neutrophil infiltration in colon tissues in a colitis model (14). In the present study, MPO activity, an important maker of neutrophils, was measured to examine the effect of UA on neutrophil infiltration. As shown in Fig. 2C, UA significantly decreased colonic MPO activity stimulated by DSS administration. Simultaneously, MPO activity was also reduced by SFZ treatment.

UA decreases the expression of pro-inflammatory cytokines. DSS induced a marked inflammatory response in the colon tissues, which was significantly improved by UA treatment. Thus, the present study examined whether UA decreases the levels of pro-inflammatory cytokines. The levels of IL-1 $\beta$ and TNF- $\alpha$ cytokines were measured using ELISA. As shown in Fig. 3, DSS administration significantly increased 

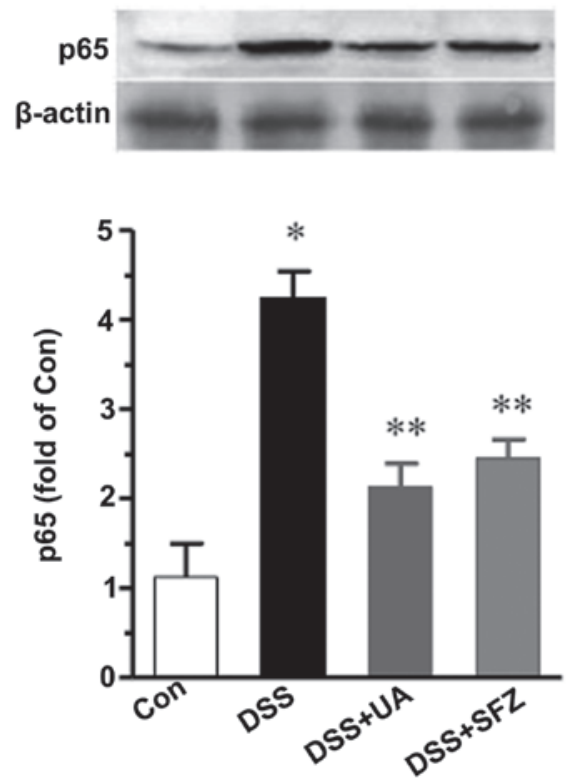

Figure 4. Effects of UA on the nuclear levels of nuclear factor- $\kappa \mathrm{B}$ p65 in colon tissues. The mice were treated with DSS for 7 days, and then with UA or SFZ for a further 7 days. The nuclear protein in the colon tissues was used to measure the levels of p65 using Western blotting. Data are presented as the mean \pm standard error of the mean $(n=5)$. ${ }^{*} \mathrm{P}<0.01$, vs. Con group ${ }^{* *} \mathrm{P}<0.05$, vs. DSS group. UA, ursolic acid; DSS, dextran sulfate sodium; SFZ, sulfasalazine; Con, control.

the serum level of IL-1 $\beta$, compared with the control group $(\mathrm{P}<0.01)$. This increase in the level of IL-1 $\beta$ was significantly decreased by UA or SFZ treatment $(\mathrm{P}<0.05)$. Similar to the results of IL-1 $\beta$, DSS administration increased the serum level of TNF- $\alpha$, which was also significantly decreased by UA or SFZ treatment.

$U A$ decreases the level of $N F-\kappa B$ p 65 . The expression levels of pro-inflammatory factors, including IL-1 $\beta$ and TNF- $\alpha$ is closely regulated by transcription factor activity, particularly $\mathrm{NF}-\kappa \mathrm{B}$. Thus, the present study examined whether UA alters the activity of $\mathrm{NF}-\kappa \mathrm{B}$ in colon tissues using Western blotting. As shown in Fig. 4, the nuclear level of NF- $\kappa$ B p65 was markedly increased by DSS, compared with the control group $(\mathrm{P}<0.01)$. Treatment with UA or SFZ resulted in a significant reduction of p65 $(\mathrm{P}<0.05)$.

\section{Discussion}

The present study aimed to evaluate the role of UA in DSS-induced colitis and the underlying mechanisms. The results showed that UA significantly improved DSS-induced colitis in mice, which was shown by improvements in body weight, stool consistency, rectal bleeding and pathologic involvement of colon tissues, and a decrease in neutrophils infiltration reflected by MPO activity. UA also decreased serum levels of IL- $1 \beta$ and TNF- $\alpha$, decreased MDA content and increased SOD activity in colon. Finally, UA downregulated DSS-stimulated nuclear expression of transcription factor $\mathrm{NF}-\kappa \mathrm{B}$. These results demonstrated a protective role of UA in experimental colitis. Antioxidant and anti-inflammatory activity may underlie the mechanisms of action.
Reactive oxygen species (ROS) is an important factor involved in ulcerative colitis. Oxidative stress and its consequent lipid peroxidation can aggravate free radical chain reactions, disrupt the integrity of intestinal mucosa barrier and activate pro-inflammatory responses. MDA, a marker of lipid peroxidative damage, and SOD, an important antioxidative enzyme, have been reported to increase and decrease in experimental colitis studies, respectively $(20,21)$. Antioxidative and anti-inflammatory treatment improved these effects on the MDA level and SOD activity in experimental ulcerative colitis $(20,21)$.

UA has showed beneficial effects on SOD activity and MDA content in various cells and animal models. UA pretreatment significantly reverses $\mathrm{H}_{2} \mathrm{O}_{2}-$ and 1-Methyl-4-phenylpyridinium-induced impairment in SOD activity, and decreases MDA formation in PC12 glial cells (22). UA increases SOD in Ehrlich ascites carcinoma tumor (23). UA has also demonstrated a neuroprotective effect on D-gal induced neurotoxicity in mice via, at least in part, increases in the activity of SOD enzymes with a reduction in MDA content (24). UA has also shown a protective effect in chronic ethanol-induced oxidative stress in the rat heart via the improvement of SOD activity (25). In the present study, SOD activity was decreased and MDA content was increased in the colon tissues of the DSS group, compared with those of the control group. This indicated that DSS administration damaged the antioxidative ability, which may subsequently cause the onset of colitis onset. This is consistent with previous reports $(26,27)$. In the present study, UA treatment for 7 days significantly improved the increased MDA content and decreased SOD activity in the colon tissues. These results suggested that antioxidative protection may be an important mechanism for UA in colitis. Furthermore, the accumulation of ROS in colon tissues, in addition to directly damaging intestinal epithelial cells, stimulates inflammatory responses to release pro-inflammatory cytokines, particularly TNF- $\alpha$ and IL-1 $\beta(28,29)$, which may cause further damage.

Ulcerative colitis is a type of inflammatory bowel disease. It is characterized by pro-inflammatory cell infiltration and pro-inflammatory cytokine release. Several cytokines, including TNF- $\alpha$, IL-1 $\beta$ and inducible NO synthetase are crucial components of these inflammatory pathways (30). In the present study, the levels of TNF- $\alpha$ and IL-1 $\beta$ were markedly upregulated during the onset of DSS-induced colitis induction. Treatment of ulcerative colitis commonly reduces inflammatory cell infiltration and the levels of serum TNF- $\alpha$ and IL-1 $\beta$ (31,32). In particular, treatment with TNF- $\alpha$ neutralizing antibody or TNF- $\alpha$ inhibitory agents significantly improveds colitis $(33,34)$. Therefore, suppression of these cytokines can offer an alternative therapy for ulcerative colitis. In the present study, when UA was applied in the DSS-administrated mice, the serum levels of TNF- $\alpha$ and IL- $1 \beta$ were significantly decreased. Given the improvement of clinical features and pathological features by UA, UA may act an inflammation alleviator in the treatment of ulcerative colitis. Of note, UA has also been found to decrease various pro-inflammatory markers, including TNF- $\alpha$, IL-1 $\beta$, IL-6, IL-17 and cyclooxygenase-2, and ameliorate inflammatory injury in experimental nephritis and hepatitis models $(35,36)$. 
In the present study, it was also demonstrated that UA significantly decreased DSS-stimulated nuclear levels of $\mathrm{NF}-\kappa \mathrm{B}$ p65 in colon tissues. NF- $\kappa \mathrm{B}$ is a dimeric transcription factor containing p50 and p65 subunits, and p65 nuclear translocation represents the activation state of $N F-\kappa B$. NF- $\kappa$ B activates the expression of several genes involved in inflammatory process, including IL-1 $\beta$ and TNF- $\alpha$. In earlier studies, UA showed a suppressive effect on NF- $\mathrm{B}$ activity in several disease models. UA protects against carbon tetrachloride-induced injury in the kidney and liver via inhibition of $\mathrm{NF}-\kappa \mathrm{B}$ activities $(35,36)$. UA also inhibits $\mathrm{T}$ cell activation and proliferation via inhibition of the NF- $\kappa \mathrm{B}$ signaling pathway (37). The administration of UA also decreases the product of lipid peroxidation and decreases the expression of $\mathrm{NF}-\kappa \mathrm{B}$ following stroke in mice (38). UA also markedly inhibits serum levels of TNF- $\alpha$, IL-6 and IL-1 $\beta$, decrease MAD levels and attenuates the protein expression of $\mathrm{NF}-\kappa \mathrm{B}$ in the lungs of LPS-treated mice (39). In terms of the gastrointestinal system, UA has a direct inhibitory effect on NF- $\kappa \mathrm{B}$ activation in intestinal epithelial cells stimulated by $\mathrm{TNF}-\alpha$, and in peritoneal macrophages stimulated by LPS (4). UA also suppresses NF- $\mathrm{BB}$ signaling in colon cancer cells (7), and other types of cancer cell $(40,41)$. In the present study, the nuclear level of NF- $\kappa \mathrm{B}$ p65 in the colon tissues of the DSS group was significantly increased, compared with that of the control group. The increased nuclear level of NF- $\kappa \mathrm{B}$ p65 was significantly reversed by UA treatment. This result, together with the direct inhibitory effects of UA on $N F-\kappa B$ signaling in intestinal epithelial cells and colon cancer cells described above, indicated that $\mathrm{NF}-\kappa \mathrm{B}$ signaling system may be important in the induction of colitis, and act an important mechanism in the protective effects of UA. Although the cause or subsequent effects of NF- $\kappa \mathrm{B}$ suppression and inflammation improvement remains to be fully elucidated, UA treatment not only decreased the nuclear expression of $N F-\kappa B$, but also downregulated the production of IL-1 $\beta$ and TNF- $\alpha$, and thereby ameliorated the severity of the colitis. Therefore, supplementation with UA may be an efficacious and promising agent in the treatment of ulcerative colitis.

In conclusion, the present results demonstrated that UA exerted a beneficial effect on DSS-induced colitis in mice. As a possible mechanism, UA improved the SOD activity, increased scavenging of oxidative-free radicals, and downregulated the levels of cytokines, including TNF- $\alpha$ and IL-1 $\beta$. The suppression of the NF- $\kappa \mathrm{B}$ transcription system may underlie the mechanism of the anti-inflammatory ability of UA.

\section{Acknowledgements}

This study was supported by the Research Startup Fund of Liaoning Medical University for Doctors and Teachers with two and more year-training abroad (grant no. Y2012B014) and the Youth Science and Technology Startup Fund of the First Affiliated Hospital of Liaoning Medical University (grant no. FY2012-17).

\section{References}

1. Furrie E, Macfarlane S, Cummings JH and Macfarlane GT: Systemic antibodies towards mucosal bacteria in ulcerative colitis and crohn's disease differentially activate the innate immune response. Gut 53: 91-98, 2004.
2. Sakthivel KM and Guruvayoorappan C: Protective effect of Acacia ferruginea against ulcerative colitis via modulating inflammatory mediators, cytokine profile and $\mathrm{NF}-\mathrm{kB}$ signal transduction pathways. J Environ Pathol Toxicol Oncol 33: 83-98, 2014.

3. Ooi CJ and Sands BE: Treatment of ulcerative colitis. Curr Opin Gastroenterol 15: 298-301, 1999.

4. Chun J, Lee C, Hwang SW, Im JP and Kim JS: Ursolic acid inhibits nuclear factor- $\mathrm{\kappa B}$ signaling in intestinal epithelial cells and macrophages and attenuates experimental colitis in mice. Life Sci 110: 23-34, 2014.

5. Baricevic D, Sosa S, Della Loggia R, Tubaro A, Simonovska B, Krasna A and Zupancic A: Topical anti-inflammatory activity of Salvia officinalis L. leaves: The relevance of ursolic acid. J Ethnopharmacol 75: 125-132, 2001.

6. Ku CM and Lin JY: Anti-inflammatory effects of 27 selected terpenoid compounds tested through modulating Th1/Th2 cytokine secretion profiles using murine primary splenocytes. Food Chem 141: 1104-1113, 2013.

7. Wang J, Liu L, Qiu H, Zhang X, Guo W, Chen W, Tian Y, Fu L, Shi D, Cheng J, et al: Ursolic acid simultaneously targets multiple signaling pathways to suppress proliferation and induce apoptosis in colon cancer cells. PLoS One 8: e63872, 2013.

8. Tang Q, Ji Q, Tang Y, Chen T, Pan G, Hu S, Bao Y, Peng W and Yin P: Mitochondrial translocation of cofilin-1 promotes apoptosis of gastric cancer BGC-823 cells induced by ursolic acid. Tumour Biol 35: 2451-2459, 2014.

9. Alqahtani A, Hamid K, Kam A, Wong KH, Abdelhak Z, Razmovski-Naumovski V, Chan K, Li KM, Groundwater PW and $\mathrm{Li}$ GQ: The pentacyclic triterpenoids in herbal medicines and their pharmacological activities in diabetes and diabetic complications. Curr Med Chem 20: 908-931, 2013.

10. D'Abrosca B, Fiorentino A, Monaco P and Pacifico S: Radical-scavenging activities of new hydroxylated ursane triterpenes from cv. Annurca apples. Chem Biodivers 2: 953-958, 2005.

11. Ali MS, Ibrahim SA, Jalil S and Choudhary MI: Ursolic acid: A potent inhibitor of superoxides produced in the cellular system. Phytother Res 21: 558-561, 2007.

12. do Nascimento PG, Lemos TL, Bizerra AM, Arriaga ÂM, Ferreira DA, Santiago GM, Braz-Filho R and Costa JG: Antibacterial and antioxidant activities of ursolic acid and derivatives. Molecules 19: 1317-1327, 2014.

13. Raphael TJ and Kuttan G: Effect of naturally occurring triterpenoids glycyrrhizic acid, ursolic acid, oleanolic acid and nomilin on the immune system. Phytomedicine 10: 483-489, 2003.

14. Malekinejad H, Shafie-Irannejad V, Hobbenaghi R, Tabatabaie SH and Moshtaghion SM: Comparative protective effect of hawthorn berry hydroalcoholic extract, atorvastatin and mesalamine on experimentally induced colitis in rats. J Med Food 16: 593-601, 2013.

15. Jang SE, Jeong JJ, Hyam SR, Han MJ and Kim DH: Ursolic acid isolated from the seed of Cornus officinalis ameliorates colitis in mice by inhibiting the binding of lipopolysaccharide to Toll-like receptor 4 on macrophages. J Agric Food Chem 62: 9711-9721, 2014.

16. Wirtz S, Neufert C, Weigmann B and Neurath MF: Chemically induced mouse models of intestinal inflammation. Nat Protoc 2: 541-546, 2007.

17. Hendrickson BA, Gokhale R and Cho JH: Clinical aspects and pathophysiology of inflammatory bowel disease. Clin Microbiol Rev 15: 79-94, 2002.

18. Aldini R, Micucci M, Cevenini M, Fato R, Bergamini C, Nanni C, Cont M, Camborata C, Spinozzi S, Montagnani M, et al: Antiinflammatory effect of phytosterols in experimental murine colitis model: Prevention, induction, remission study. PLoS One 9: e108112, 2014.

19. Wang L, Wang J, Wang Y, Fu Q, Lei YH, Nie ZY, Qiu J and Bao TY: Protective effect of exogenous matrix metalloproteinase-9 on chronic renal failure. Exp Ther Med 7: 329-334, 2014.

20. Tahan G, Aytac E, Aytekin H, Gunduz F, Dogusoy G, Aydin S, Tahan V and Uzun H: Vitamin E has a dual effect of anti-inflammatory and antioxidant activities in acetic acid-induced ulcerative colitis in rats. Can J Surg 54: 333-338, 2011.

21. Zhou YH, Yu JP, Liu YF, Teng XJ, Ming M, Lv P, An P, Liu SQ and Yu HG: Effects of Ginkgo biloba extract on inflammatory mediators (SOD, MDA, TNF-alpha, NF-kappaBp65, IL-6) in TNBS-induced colitis in rats. Mediators Inflamm 5: 92642, 2006. 
22. Tsai SJ and Yin MC: Antioxidative and anti-inflammatory protection of oleanolic acid and ursolic acid in PC12 cells. J Food Sci 73: H174-H178, 2008.

23. Saraswati S, Agrawal SS and Alhaider AA: Ursolic acid inhibits tumor angiogenesis and induces apoptosis through mitochondrial-dependent pathway in Ehrlich ascites carcinoma tumor. Chem Biol Interact 206: 153-165, 2013.

24. Lu J, Zheng YL, Wu DM, Luo L, Sun DX and Shan Q: Ursolic acid ameliorates cognition deficits and attenuates oxidative damage in the brain of senescent mice induced by D-galactose. Biochem Pharmacol 74: 1078-1090, 2007.

25. Saravanan R and Pugalendi V: Impact of ursolic acid on chronic ethanol-induced oxidative stress in the rat heart. Pharmacol Rep 58: 41-47, 2006

26. Zhao J, Hong T, Dong M, Meng Y and Mu J: Protective effect of myricetin in dextran sulphate sodium-induced murine ulcerative colitis. Mol Med Rep 7: 565-570, 2013.

27. Farombi EO, Adedara IA, Awoyemi OV, Njoku CR, Micah GO, Esogwa CU, Owumi SE and Olopade JO: Dietary protocatechuic acid ameliorates dextran sulphate sodium-induced ulcerative colitis and hepatotoxicity in rats. Food Funct 7: 913-921, 2016.

28. Lee JH, Lee B, Lee HS, Bae EA, Lee H, Ahn YT, Lim KS, Huh CS and Kim DH: Lactobacillus suntoryeus inhibits pro-inflammatory cytokine expression and TLR-4-linked NF-kappaB activation in experimental colitis. Int J Colorectal Dis 24: 231-237, 2009.

29. Zhang DK, Yu JJ, Li YM, Wei LN, Yu Y,Feng YH and Wang X: A Picrorhiza kurroa derivative, picroliv, attenuates the development of dextran-sulfate-sodium-induced colitis in mice. Mediators Inflamm 2012: 751629, 2012

30. Oishi M, Tokuhara K, Miki H, Tanaka Y, Yamaki S, Kaibori M, Yoshizawa K, Yuri T, Yoshigai E, Nishizawa M, et al: Temporal and spatial dependence of inflammatory biomarkers and suppression by fluvastatin in dextran sodium sulfate-induced rat colitis model. Dig Dis Sci 59: 2126-2135, 2014.

31. Zhang ZL, Fan HY, Yang MY, Zhang ZK and Liu K: Therapeutic effect of a hydroxynaphthoquinone fraction on dextran sulfate sodium-induced ulcerative colitis. World J Gastroenterol 20 $15310-15318,2014$
32. Fakhoury M, Coussa-Charley M, Al-Salami H, Kahouli I and Prakash S: Use of artificial cell microcapsule containing thalidomide for treating TNBS-induced Crohn's disease in mice. Curr Drug Deliv 11: 146-153, 2014.

33. Dharmani $P$, Leung $P$ and Chadee K: Tumor necrosis factor- $\alpha$ and Muc2 mucin play major roles in disease onset and progression in dextran sodium sulphate-induced colitis. PLoS One 6: e25058, 2011.

34. Lv R, Qiao W, Wu Z, Wang Y, Dai S, Liu Q and Zheng X: Tumor necrosis factor alpha blocking agents as treatment for ulcerative colitis intolerant or refractory to conventional medical therapy: A meta-analysis. PLoS One 9: e86692, 2014.

35. Ma JQ, Ding J, Xiao ZH and Liu CM: Ursolic acid ameliorates carbon tetrachloride-induced oxidative DNA damage and inflammation in mouse kidney by inhibiting the STAT3 and NF- $\kappa \mathrm{B}$ activities. Int Immunopharmacol 21: 389-395, 2014.

36. Ma JQ, Ding J, Zhang L and Liu CM: Ursolic acid protects mouse liver against $\mathrm{CCl} 4$-induced oxidative stress and inflammation by the MAPK/NF- $\kappa$ B pathway. Environ Toxicol Pharmacol 37: 975-983, 2014

37. Zeng G, Chen J, Liang QH, You WH, Wu HJ and Xiong XG: Ursolic acid inhibits T-cell activation through modulating nuclear factor- $\kappa \mathrm{B}$ signaling. Chin J Integr Med 18: 34-39, 2012.

38. Li L, Zhang X, Cui L, Wang L, Liu H, Ji H and Du Y: Ursolic acid promotes the neuroprotection by activating Nrf2 pathway after cerebral ischemia in mice. Brain Res 1497: 32-39, 2013.

39. Chen X, Wan Y, Zhou T, Li J and Wei Y: Ursolic acid attenuates lipopolysaccharide-induced acute lung injury in a mouse model. Immunotherapy 5: 39-47, 2013.

40. Gai L, Cai N, Wang L, Xu X and Kong X: Ursolic acid induces apoptosis via Akt/NF- $\kappa \mathrm{B}$ signaling suppression in T24 human bladder cancer cells. Mol Med Rep 7: 1673-1677, 2013.

41. Liu K, Guo L, Miao L, Bao W, Yang J, Li X, Xi T and Zhao W: Ursolic acid inhibits epithelial-mesenchymal transition by suppressing the expression of astrocyte-elevated gene-1 in human nonsmall cell lung cancer A549 cells. Anticancer Drugs 24: 494-503, 2013. 